\title{
Patterns of epidural progression following postoperative spine stereotactic body radiotherapy: implications for clinical target volume delineation
}

\author{
Michael W. Chan, MD, Isabelle Thibault, MD, ${ }^{2}$ Eshetu G. Atenafu, MSc, ${ }^{3}$ Eugene Yu, MD, ${ }^{4}$ \\ B. C. John Cho, MD, PhD, ${ }^{5}$ Daniel Letourneau, PhD, ${ }^{5}$ Young Lee, PhD, ${ }^{2}$ Albert Yee, MD, ${ }^{6}$ \\ Michael G. Fehlings, MD, PhD, ${ }^{7}$ and Arjun Sahgal, MD ${ }^{2,5}$
}

\begin{abstract}
Departments of ${ }^{1}$ Medical Imaging and ${ }^{4}$ Radiology, University Health Network, University of Toronto; ${ }^{2}$ Department of Radiation Oncology, Odette Cancer Centre, and ${ }^{6}$ Division of Orthopaedic Surgery, Department of Surgery, Sunnybrook Health Sciences Centre, University of Toronto; Departments of ${ }^{3}$ Biostatistics and ${ }^{5}$ Radiation Oncology, Princess Margaret Cancer Centre, University of Toronto; and 'Division of Neurosurgery, Department of Surgery, Toronto Western Hospital, University of Toronto, Ontario, Canada
\end{abstract}

OBJECT The authors performed a pattern-of-failure analysis, with a focus on epidural disease progression, in patients treated with postoperative spine stereotactic body radiotherapy (SBRT).

METHODS Of the 70 patients with 75 spinal metastases (cases) treated with postoperative spine SBRT, there were 26 cases of local disease recurrence and 25 cases with a component of epidural disease progression. Twenty-four of the 25 cases had preoperative epidural disease with subsequent epidural disease progression, and this cohort was the focus of this epidural-specific pattern-of-failure investigation. Preoperative, postoperative, and follow-up MRI scans were reviewed, and epidural disease was characterized based on location according to a system in which the vertebral anatomy is divided into 6 sectors, with the anterior compartment comprising Sectors 1,2, and 6, and the posterior compartment comprising Sectors 3,4 , and 5 .

RESULTS Patterns of epidural progression are reported specifically for the 24 cases with preoperative epidural disease and subsequent epidural progression. Epidural disease progression within the posterior compartment was observed to be significantly lower in those with preoperative epidural disease confined to the anterior compartment than in those with preoperative epidural disease involving both anterior and posterior compartments ( $56 \%$ vs $93 \%$, respectively; $p=0.047$ ). In a high proportion of patients with epidural disease progression, treatment failure was found in the anterior compartment, including both those with preoperative epidural disease confined to the anterior compartment and those with preoperative epidural disease involving both anterior and posterior compartments ( $100 \%$ vs. $73 \%$, respectively). When epidural disease was confined to the anterior compartment on the preoperative and postoperative MRIs, no epidural disease progression was observed in Sector 4 , which is the most posterior sector. Postoperative epidural disease characteristics alone were not predictive of the pattern of epidural treatment failure.

CONCLUSIONS Reviewing the extent of epidural disease on preoperative MRI is imperative when planning postoperative SBRT. When epidural disease is confined to the anterior epidural sectors pre- and postoperatively, covering the entire epidural space circumferentially with a prophylactic "donut" distribution may not be needed.

http://thejns.org/doi/abs/10.3171/2015.6.SPINE15294

KEY WORDS spinal metastases; malignant epidural spinal cord compression; postoperative radiosurgery; stereotactic body radiotherapy; local recurrence; patterns of failure; epidural disease; spine radiosurgery; oncology

ABBREVIATIONS CTV = clinical target volume; ISRC = International Spine Radiosurgery Consortium; LC = local control; OS = overall survival; PTV = planning tumor volume; $S B R T=$ stereotactic body radiotherapy; $V_{\text {rec }}=$ recurrent tumor volume .

SUBMITTED March 12, 2015. ACCEPTED June 11, 2015.

INCLUDE WHEN CITING Published online December 18, 2015; DOI: 10.3171/2015.6.SPINE15294. 
$\mathrm{S}$ TEREOTACTIC body radiotherapy (SBRT) allows the targeting of high doses of radiation with steep dose gradients, so that organs at risk like the spinal cord can be intentionally spared in order to maintain safety and conformality. ${ }^{10,11}$ The primary goal of spine SBRT is to deliver locally "curative" doses of radiation in just a few fractions to maximize both local control and pain control. ${ }^{13}$ There is growing evidence demonstrating the efficacy of spine SBRT, with excellent local control and pain control rates in patients receiving de novo treatment, retreatment after prior radiation, and, more recently, postoperative adjuvant therapy following resection. ${ }^{16,15,16}$

Postoperative spine SBRT is growing in practice, as it fits with the rationale that if we are going to subject a patient to a major operation, then we should deliver an effective and philosophically equivalent adjuvant therapy intended to maximize local control. ${ }^{10}$ Imaging-based local control rates after conventional palliative radiation therapy have not been well defined. Based on the few studies that have been reported, these control rates are modest at best and represent an area for improvement. ${ }^{5,8}$ In the limited literature on postoperative spine SBRT, the rates of local control are highly promising, as 1-year local control rates of approximately $80 \%$ to $90 \%$ have been reported..$^{1,6,7}$

Surgery for spinal metastases is often performed for decompression of the spinal cord and involves resection of epidural disease. However, a major area of uncertainty is related to the most appropriate clinical target volume (CTV) for postoperative spine SBRT. Specific to intact metastases, the International Spine Radiosurgery Consortium (ISRC) has defined CTV-contouring practice; ${ }^{4}$ however, there is no such guideline for patients undergoing SBRT postoperatively. The fundamental issues include whether to treat only residual postoperative epidural disease as evident on postoperative MRI; whether to account for all areas of epidural extension based on preoperative MRI imaging of the CTV; whether to put the CTV margins craniocaudally, as epidural disease does not only infiltrate circumferentially into the axial plane; and whether to simply cover the entire epidural space circumferentially and prophylactically, per a "donut" distribution (Fig. 1), in order to be as safe as possible whenever epidural disease is present. Given that progression within the epidural space has been reported as the most common pattern of treatment failure,,$^{10}$ the rationale for the donut distribution is to reduce the risk of epidural progression as much as possible because the consequence of progression is cord compression that puts the patient at risk for further operative management and radiation. This has been common practice at the University of Toronto, per our previously published report specific to postoperative spine SBRT. ${ }^{1}$

The purpose of this investigation was to perform, as an extension of our previous outcome analysis, ${ }^{1}$ a detailed pattern-of-failure analysis focused on the epidural space. In addition, we sought to determine if any relationship with epidural progression is observable that influences CTV delineation based on both the preoperative and postoperative epidural disease characteristics.

\section{Methods}

The primary objective of this study was to investigate the patterns of local treatment failure within the epidural space following postoperative spine SBRT. The outcomes and technique applied to this cohort have been previously reported. ${ }^{1}$ In that report, we also provided a detailed summary of the dosimetric outcomes and doses to the spinal cord and thecal sac (i.e., critical neural tissues). Of note regarding the original series, the only predictors for local control were the epidural disease grade and prescribed dose, and none of the dosimetric outcomes-including the dose to the critical neural tissues - were predictive. Therefore, we designed this study to anatomically determine the patterns of progression within the epidural space, as the dose to the critical neural structures was not predictive.

Of the original 80 patients previously reported upon with institutional research ethics board approval, only those with preoperative, postoperative, and post-SBRT follow-up MR images available for central review were included in this pattern-of-failure study. The final cohort consisted of 70 patients with 75 spinal SBRT targets treated, and the reason for excluding the 10 patients from our previous report was the lack of preoperative MRI available for central review. Each preoperative, postoperative, and follow-up spine MRI study was reviewed by both a neuroradiologist and radiation oncologist. The presence of epidural disease was categorized by location and mapped according to a system in which the vertebral anatomy is divided into 6 sectors, as previously described by the ISRC $^{4}$ (Fig. 2). In addition, the grade of epidural disease, according to the Bilsky system, ${ }^{3}$ was determined within each sector. Disease progression within an ISRC sector was defined as any increase in epidural tumor dimension compared with the baseline treatment-planning postoperative MRI, or new epidural disease if previously undocumented. Local recurrences were classified as "in-field" if $95 \%$ or more of the recurrent tumor volume $\left(\mathrm{V}_{\text {rec }}\right)$ was within the planning tumor volume (PTV); "marginal" if $20 \%$ to $94 \%$ of $\mathrm{V}_{\text {rec }}$ was within the PTV; or "out-of-field" if less than $20 \%$ of $\mathrm{V}_{\text {rec }}$ was within the PTV.

Figure 2 illustrates the ISRC system. ${ }^{4}$ In brief, Sector 1 refers to the vertebral body and associated anterior epidural space; Sectors 2 and 6 refer to the right and left pedicles and associated epidural spaces, respectively; Sectors 3 and 5 refer to the right and left transverse processes and laminae and associated epidural spaces, respectively; and Sector 4 refers to the spinous process and associated epidural space. Although the anatomical nomenclature stipulates the vertebral body to be "anterior" and the remaining structures (pedicles, transverse process, laminae, spinous process) to form the "posterior" elements, for the purposes of this study we classified Sectors 1, 2, and 6 as the "anterior" compartment and Sectors 3, 4, and 5 as the "posterior" compartment. This modified compartment division was felt to be more clinically relevant. Based on this anatomical classification system, we evaluated the patterns of epidural failure following SBRT based on 1) the involved ISRC sectors (Sectors 1-6) on preoperative and postoperative MRI, 2) the sum of the ISRC sectors involved, and 3) the worst grade of the epidural disease according to Bilsky criteria. $^{3}$

\section{Statistical Analysis}

Descriptive statistics were used to assess patient demo- 

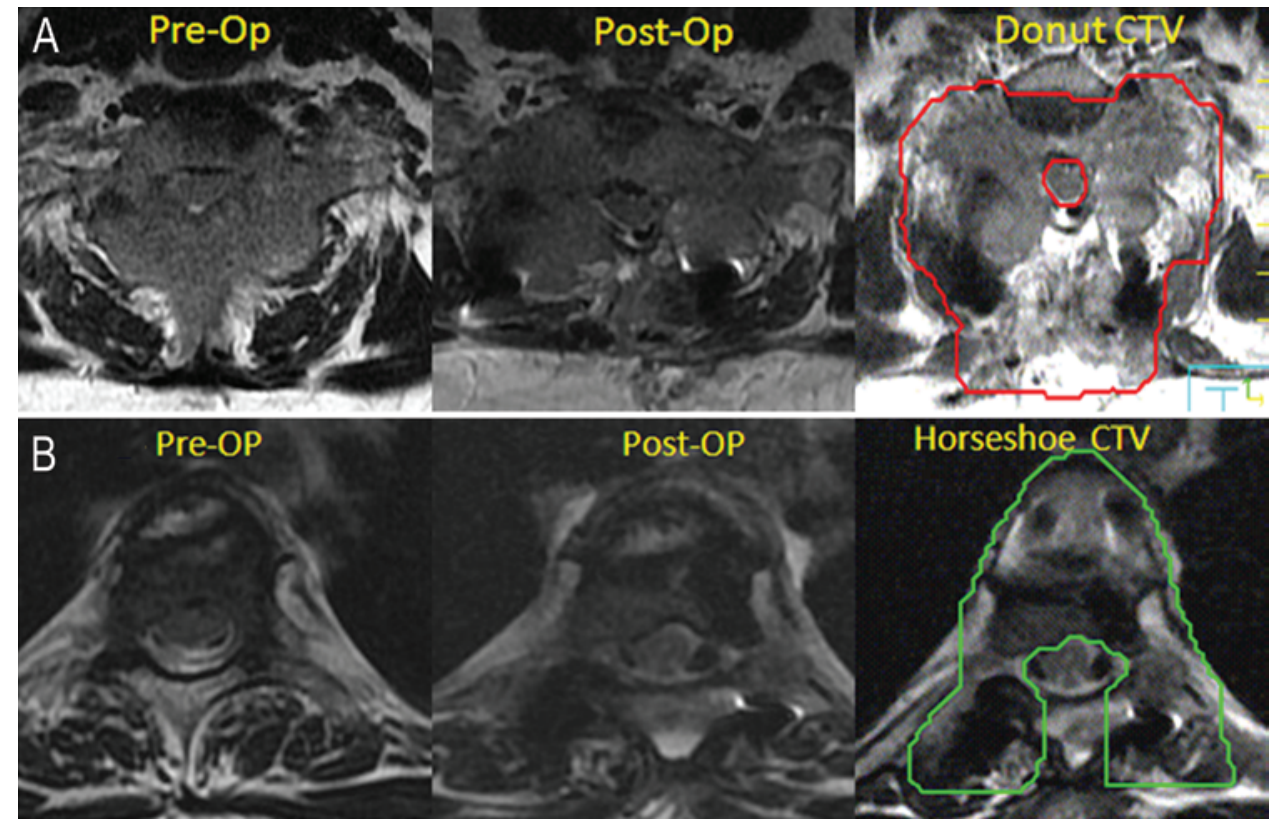

FIG. 1. A: MR images obtained in a patient with lung cancer and C-7 metastases involving the ISRC anterior and posterior bony segments and epidural sectors circumferentially. Postoperatively, the patient underwent decompression, and the posterior elements were resected with no residual epidural disease in Sector 4. The CTV applied was a donut distribution, given that all ISRS sectors were involved preoperatively. B: MR images obtained in a breast cancer patient with T-3 metastases involving the vertebral body and bilateral pedicles and epidural disease confined to the anterior ISRC sectors (1, 2, and 6). Postoperatively, the posterior elements were removed and residual epidural disease in the same anterior sectors is observed. A horseshoe CTV was applied, given the lack of epidural disease on preoperative and postoperative MRI in the posterior sectors (Sectors 3, 4, and 5) and no residual bony or soft-tissue disease within those posterior sectors. Figure is available in color online only.

graphics, tumor characteristics, and covariates of interest. Categorical variables were expressed as count and proportions, whereas continuous variables were expressed as the mean \pm standard deviation or median and range. Time to local control (LC) was evaluated in months from the start of SBRT up to the date of local failure, death, or the last follow-up. The cumulative incidence of local recurrence was calculated using Fine and Gray's competing risk methods and considered death as a competing risk. ${ }^{9}$ Overall survival (OS) was calculated using the Kaplan-Meier method. The Fisher exact test was used to determine if any association existed between the initial epidural tumoral characteristics (according to both the preoperative and postoperative treatment-planning MRIs) and the pattern of disease progression within the ISRC sectors noted on the first MRI scan with evidence of local disease progression. All $\mathrm{p}$ values were 2 sided, and $\mathrm{p}<0.05$ was considered to indicate a significantly different result. Data analysis was performed using SAS (version 9.3; SAS Institute, Inc.) and open source statistical software (R, version 3.0.3; R Foundation for Statistical Computing).

\section{Results}

\section{Patients and Treatment Characteristics}

A total of 70 patients with 75 spinal metastases treated
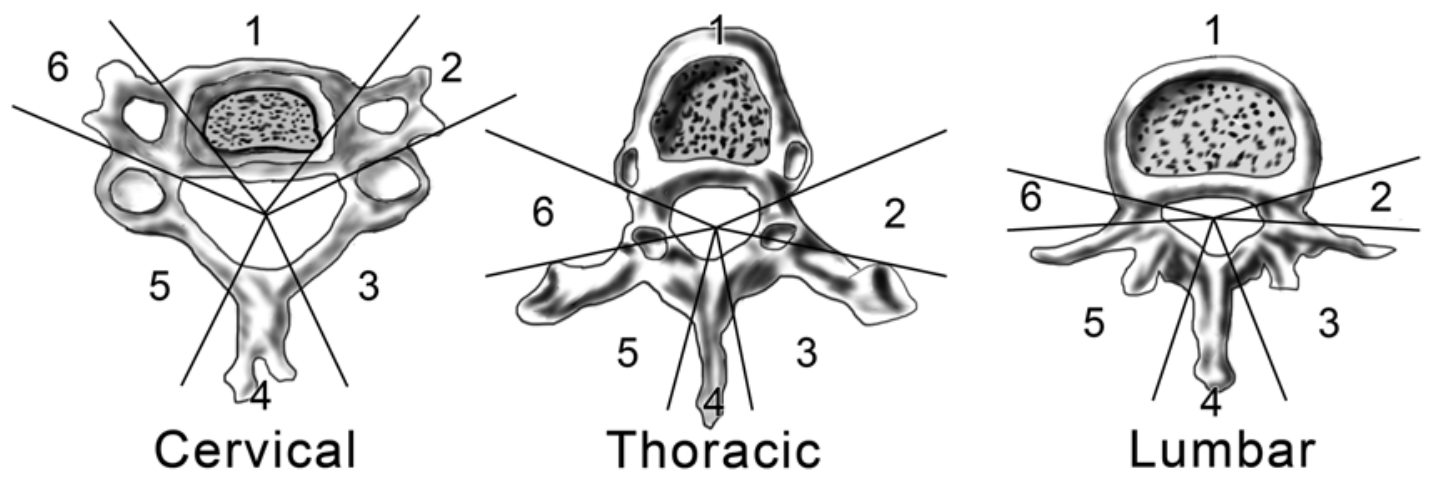

FIG. 2. The IRSC system divides each cervical, thoracic, and lumbar vertebra into 6 anatomical sectors: Sector 1 refers to the vertebral body and associated anterior epidural space; Sectors 2 and 6 refer to the left and right pedicles and associated epidural spaces, respectively; Sectors 3 and 5 refer to the left and right transverse processes, laminae, and associated epidural spaces, respectively; and Sector 4 refers to the spinous process and associated epidural space. 
with postoperative SBRT were evaluated for this analysis. Of these patients, 35 of $70(50 \%)$ were female patients and the median age was 59 years (range 18-81 years). The tumor and treatment characteristics are summarized in Table 1 for the entire cohort. The median follow-up was 10.8 months (range 0.8-49.2 months). The 1-year OS and
LC rates were $52.2 \%(95 \%$ CI $40.1 \%-62.9 \%)$ and $79.6 \%$ (95\% CI 65.3\%-93.8\%), respectively. Of the 75 treated tumors (cases), 26 (34.7\%) exhibited evidence of progression on follow-up MRI. The baseline/treatment characteristics for the 26 local failures are also summarized separately in Table 1, which includes the type of surgery performed.

TABLE 1. Baseline tumor and treatment characteristics of the 70 patients and 75 spinal tumors treated with postoperative SBRT

\begin{tabular}{|c|c|c|}
\hline Characteristics & All Tumors $(n=75)$ & Local Failures $(n=26)$ \\
\hline \multicolumn{3}{|l|}{ Primary cancer type } \\
\hline Breast & $12(16.0 \%)$ & $3(11.5 \%)$ \\
\hline Lung & $10(13.3 \%)$ & $3(11.5 \%)$ \\
\hline Thyroid & $10(13.3 \%)$ & $4(15.4 \%)$ \\
\hline Kidney & $6(8.0 \%)$ & $1(3.9 \%)$ \\
\hline Hepatocellular & $5(6.7 \%)$ & $3(11.5 \%)$ \\
\hline Other & $32(42.7 \%)$ & $12(46.2 \%)$ \\
\hline \multicolumn{3}{|l|}{ Spinal level } \\
\hline Cervical & $11(14.7 \%)$ & $3(11.5 \%)$ \\
\hline Thoracic & $44(58.7 \%)$ & $18(69.2 \%)$ \\
\hline Lumbar-sacrum & $20(26.6 \%)$ & $5(19.2 \%)$ \\
\hline Paraspinal tumor extension & $58(77.3 \%)$ & $22(84.6 \%)$ \\
\hline Prior radiation exposure & $26(34.7 \%)$ & $9(34.6 \%)$ \\
\hline Donut distribution & $68(91 \%)$ & $24(92 \%)$ \\
\hline \multicolumn{3}{|l|}{ Preop epidural Bilsky grade } \\
\hline 0 & $7(9.3 \%)$ & $1(3.9 \%)$ \\
\hline $1 a, 1 b$, or $1 c$ & $28(37.3 \%)$ & $11(42.3 \%)$ \\
\hline 2 or 3 & $40(53.3 \%)$ & $14(53.9 \%)$ \\
\hline \multicolumn{3}{|l|}{ Preop tumor location w/in epidural space } \\
\hline Confined to anterior epidural compartment or no preexisting epidural disease & $29(38.7 \%)$ & $10(38.5 \%)$ \\
\hline $\begin{array}{l}\text { Posterior epidural compartment w/ anterior epidural compartment involve- } \\
\text { ment }\end{array}$ & $46(61.3 \%)$ & $16(61.5 \%)$ \\
\hline Confined to the posterior epidural compartment & $0(0 \%)$ & $0(0 \%)$ \\
\hline$\geq 4$ sectors containing epidural disease preop & $39(52.0 \%)$ & $15(57.7 \%)$ \\
\hline Median no. of epidural sectors involved & 4 (range $0-6$ ) & 4 (range $0-6$ ) \\
\hline \multicolumn{3}{|l|}{ Total dose and no. of fractions } \\
\hline 20 Gy/1 fraction & $3(4.0 \%)$ & $1(3.9 \%)$ \\
\hline $18-26$ Gy/2 fractions & $31(41.3 \%)$ & $9(34.6 \%)$ \\
\hline $18-40$ Gy/3-5 fractions & $41(54.7 \%)$ & $16(61.5 \%)$ \\
\hline \multicolumn{3}{|l|}{ Surgical details } \\
\hline Stabilization procedure alone & $6(8.0)$ & $1(3.9 \%)$ \\
\hline Decompression w/o stabilization & $20(26.7 \%)$ & $7(26.9 \%)$ \\
\hline Decompression w/ instrumented stabilization & $49(65.3 \%)$ & $18(69.2 \%)$ \\
\hline \multicolumn{3}{|l|}{ Postop epidural Bilsky grade } \\
\hline 0 & $15(20.0 \%)$ & $4(15.4 \%)$ \\
\hline $1 \mathrm{a}, 1 \mathrm{~b}$, or $1 \mathrm{c}$ & $54(72.0 \%)$ & $18(69.2 \%)$ \\
\hline 2 or 3 & $6(8.0 \%)$ & $4(15.4 \%)$ \\
\hline \multicolumn{3}{|l|}{ Postop tumor location w/in epidural space } \\
\hline Confined to anterior epidural compartment or no preexisting epidural disease & $55(73.3 \%)$ & $21(80.8 \%)$ \\
\hline $\begin{array}{l}\text { Posterior epidural compartment w/ anterior epidural compartment involve- } \\
\text { ment }\end{array}$ & $20(26.7 \%)$ & $5(19.2 \%)$ \\
\hline Confined to posterior epidural compartment & $0(0 \%)$ & $0(0 \%)$ \\
\hline$\geq 4$ sectors containing epidural disease on postop MRI & $13(17.3 \%)$ & $4(15.4 \%)$ \\
\hline
\end{tabular}


Most spinal segments (24 of 26; 92\%) were treated with a circumferential donut-shaped CTV (Fig. 1). With respect to the 2 patients treated with a nondonut horseshoe CTV (Fig. 1), preoperative disease was confined to the anterior compartment (Sectors 1, 2, and 6) and, upon failure, epidural disease progression was also confined to the anterior compartment. Twenty-five cases ( 25 of 26 ; $96 \%$ ) demonstrated epidural disease progression and 1 case demonstrated local failure limited to the bone. Of the 25 cases with epidural disease progression, 1 case did not have preoperative epidural disease, resulting in 24 cases with preoperative epidural disease and subsequent epidural disease progression. This group of 24 cases is the focus of the pattern of epidural disease progression analysis. A detailed summary of the epidural tumor characteristics for these 24 cases before surgery, postsurgery, and at the time of failure is presented in Table 2.

\section{Patterns of Failure}

Tumor progression was observed within the epidural space in 25 of $26(96 \%)$ failures, bone in 19 of $26(73 \%)$ failures, and paraspinal tissues in 10 of 26 (38\%) failures. With respect to local failure in the bone and paraspinal tissues, 5 of $26(19 \%)$ cases demonstrated marginal recurrence, 19 of $26(73 \%)$ had a component of in-field recurrence, and none were out-of-field recurrences. With respect to the gross patterns of epidural progression, 1 of $26(4 \%)$ progressed circumferentially within the epidural space (all sectors), 19 of 26 (73\%) involved $\geq 4$ epidural sectors, and 14 of $26(54 \%)$ lesions were high-grade lesions at the time of progression (Bilsky Grade 2 or 3). With the exception of 1 case, all cases of failure had preexisting epidural disease. For the 1 case with no epidural disease on the preoperative or postoperative MRI, the bony tumor was confined to the vertebral body and epidural progression was confined to the anterior compartment involving Sec- tors 1,2 , and 6 . This case was excluded from the epidural disease pattern-of-failure analysis.

Table 2 provides a detailed summary of the 24 cases with preoperative epidural disease and subsequent epidural disease progression. In particular, Table 2 illustrates differences in epidural disease distribution by ISRC sector between those with preoperative epidural disease confined to the anterior compartment (Sectors 1,2, and 6) and those with preoperative epidural disease involving the anterior and posterior compartments (Sectors 1, 2, or 6 and 5, 4, or 3) based on the preoperative MRI, postoperative MRI, and first follow-up MRI demonstrating epidural disease progression. No patient had preoperative epidural disease confined to the posterior epidural space alone (Sectors 3, 4, and 5).

When epidural disease was confined to the anterior compartment on preoperative MRI ( 9 of 24 patients; $38 \%$ ), the anterior compartment was also involved in 8 of $9(89 \%)$ cases on postoperative imaging and in 9 of $9(100 \%)$ cases upon progression. Progression was most often observed (7 of 9 patients; $78 \%$ ) in the immediate anterior sector and left lateral sector (Sectors 1 and 6, respectively), followed by the right lateral epidural space (Sector 2 in 6 of 9 patients; 67\%). Disease location according to the preoperative and postoperative MR images indicated a similar profile with respect to the proportions of involvement in Sectors 1,2, and 6. New epidural disease within the previously uninvolved posterior epidural space was observed in 5 of these 9 cases (56\%). With respect to posterior sectors exhibiting epidural disease progression, equivalent proportions of involvement were observed within the right and left posterolateral epidural spaces (Sectors 3 and 5); no progression was observed in Sector 4 , which is diametrically opposed to Sector 1 (Fig. 3).

When epidural disease involved both the anterior and posterior compartments preoperatively (15 of 24 patients; 63\%) (Table 2), Sectors 1 and 2 were involved in 15 of

TABLE 2. Location of preoperative epidural disease, postoperative epidural disease, and disease progression by epidural region in the 24 treated spinal segments (cases) with preoperative epidural disease and subsequent epidural disease progression

\begin{tabular}{|c|c|c|c|c|}
\hline \multirow[b]{2}{*}{ Variable } & \multicolumn{4}{|c|}{ Sector; No. of Cases (\%) } \\
\hline & $\begin{array}{c}\text { Preop Epidural } \\
\text { Sector Involvement }\end{array}$ & $\begin{array}{c}\text { Postop Epidural } \\
\text { Sector Involvement }\end{array}$ & $\begin{array}{l}\text { Anterior Compartment \& Sector } \\
\text { Involvement on Progression }\end{array}$ & $\begin{array}{l}\text { Posterior Compartment \& Sector } \\
\text { Involvement on Progression }\end{array}$ \\
\hline \multirow{4}{*}{$\begin{array}{l}\text { Preop epidural disease confined to an- } \\
\text { terior compartment }(9 / 24 ; 38 \%)\end{array}$} & $1 ; 8 / 9(88.9 \%)$ & $1 ; 7 / 9(77.8 \%)$ & $9 / 9(100 \%)$ & $5 / 9(55.6 \%)$ \\
\hline & $2 ; 5 / 9(55.6 \%)$ & $2 ; 5 / 9(55.6 \%)$ & $1 ; 7 / 9(77.8 \%)$ & $2 ; 3 / 5(60 \%)$ \\
\hline & $6 ; 7 / 9(77.8 \%)$ & $6 ; 6 / 9(66.7 \%)$ & $2 ; 6 / 9(66.7 \%)$ & $4 ; 0 / 5(0 \%)$ \\
\hline & & & $6 ; 7 / 9(77.8 \%)$ & $5 ; 3 / 5(60 \%)$ \\
\hline \multirow{6}{*}{$\begin{array}{l}\text { Preop epidural disease involving ante- } \\
\text { rior \& posterior compartments } † \\
(15 / 24 ; 63 \%)\end{array}$} & $1 ; 15 / 15(100 \%)$ & $1 ; 14 / 15(93.3 \%)$ & $11 / 15(73.3 \%)$ & $14 / 15(93.3 \%)$ \\
\hline & $2 ; 15 / 15(100 \%)$ & $2 ; 14 / 15(93.3 \%)$ & $1 ; 9 / 11(81.8 \%)$ & $3 ; 10 / 14(71.4 \%)$ \\
\hline & $6 ; 14 / 15(93.3 \%)$ & $6 ; 12 / 15(80 \%)$ & $2 ; 8 / 11(72.7 \%)$ & $4 ; 4 / 14(28.6 \%) \dagger$ \\
\hline & $3 ; 14 / 15(93.3 \%)$ & $3 ; 3 / 15(20 \%)$ & 6; 9/11 (81.8\%) & $5 ; 8 / 14(57.1 \%)$ \\
\hline & $4 ; 4 / 15(26.7 \%) \dagger$ & $4 ; 0 / 15(0 \%)$ & & \\
\hline & $5 ; 8 / 15(53.3 \%)$ & $5 ; 1 / 15(6.7 \%)$ & & \\
\hline
\end{tabular}

* Cases refers to spinal segments treated with SBRT.

$\dagger$ Only 2 of the cases exhibiting epidural disease progression in Sector 4 demonstrated epidural disease in Sector 4 on preoperative MRI. 


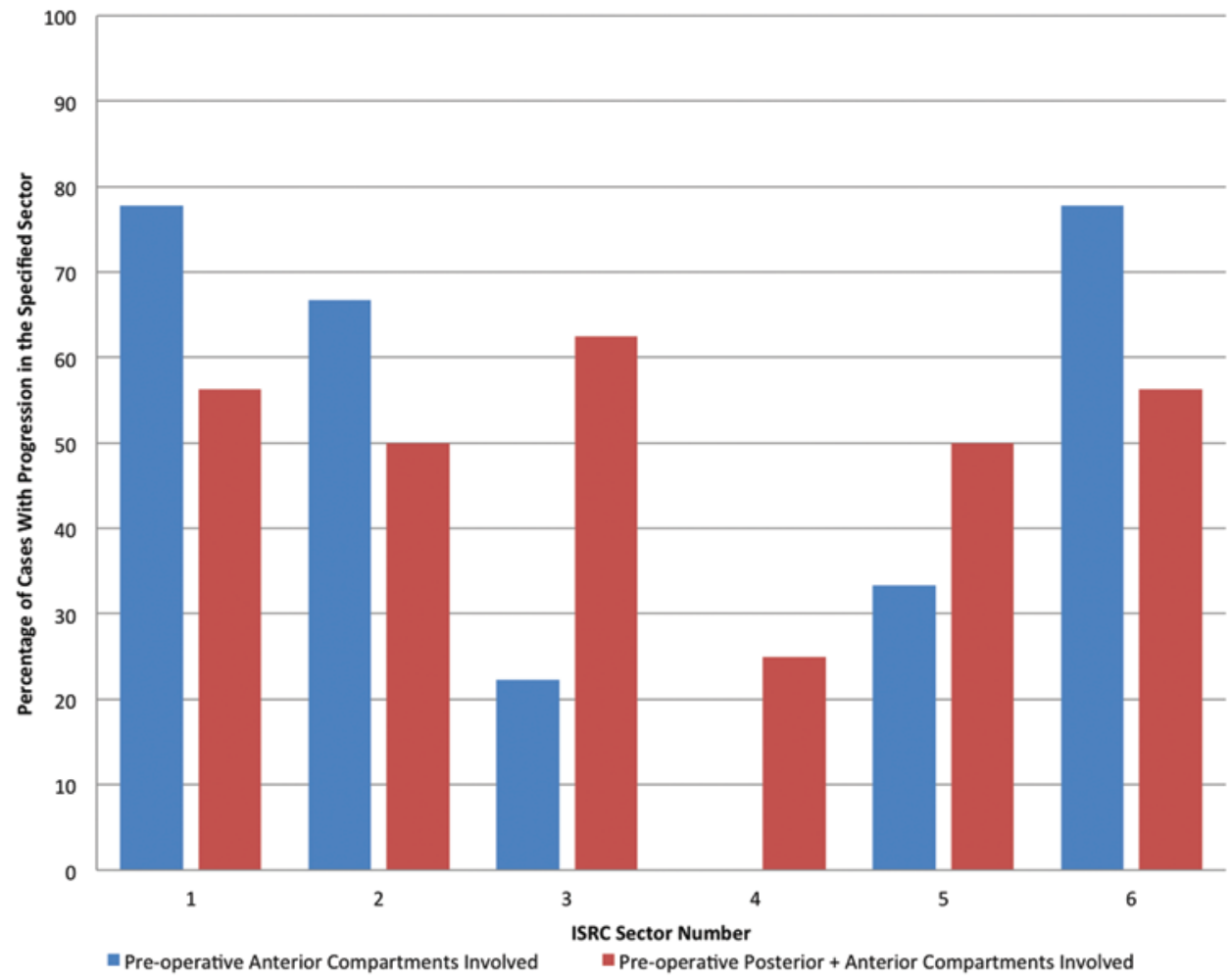

FIG. 3. The proportions of spinal metastases with epidural tumor recurrence distributed within each of the 6 ISRC vertebral sectors according to the 9 recurrent metastases with preoperative epidural disease confined to the anterior compartment (Sectors 1,2, or 6) alone, and the 15 recurrent metastases with preoperative posterior compartment and anterior involvement (Sectors 3, 4, or 5, and 1,2 , or 6 , respectively). Figure is available in color online only.

15 cases, and Sector 6 in 14 of 15 cases. Sectors 3 and 5 were involved in 14 of $15(93.3 \%)$ and 8 of $15(53.3 \%)$ cases, respectively, and Sector 4 in 4 of 15 (26.7\%) cases. Postoperatively, persistent epidural disease was observed in the anterior compartment in the majority of cases $(14$ of 15 patients; 93.3\%). Within the posterior compartment, a reduction in the number of cases with epidural disease involvement was observed (4 of 15 cases; $27 \%$; confined to Sectors 5 or 3). No epidural disease remained in Sector 4 postoperatively. At the time of failure, epidural disease progression was observed in the anterior compartment in 11 of $15(73.3 \%)$ cases, and in the posterior compartment for 14 of $15(93.3 \%)$ cases. With respect to the anterior sectors, approximately equal proportions of disease failure were observed in Sectors 1, 2, and 6. With respect to posterior-compartment disease failure, Sectors 3 and 5 were most often involved (10 of 14 [71.4\%] and 8 of 14 [57.1\%] cases, respectively), and Sector 4 was involved in 4 cases $(28.6 \%)$

The proportion of cases with posterior-compartment epidural disease failure was significantly lower when the preoperative disease was confined to the anterior compartment $(\mathrm{p}=0.047)$. With respect to the Bilsky grade, high-grade preoperative disease (Bilsky Grade 1C, 2, and 3 ) was associated with greater failure within the posterior epidural compartment sectors $(p=0.018)$. Postoperative Bilsky grade did not demonstrate any association with the location of epidural disease progression. No association was found between the degree of preoperative disease, i.e., the total (cumulative) number of ISRC sectors involved, and the risk of epidural disease progression following $\operatorname{SBRT}(\mathrm{p}=0.72)$.

\section{Discussion}

We present the most detailed epidural pattern-of-failure analysis to date in patients with preoperative epidural disease who underwent surgery, were treated with postoperative spine SBRT, and subsequently suffered treatment failure with epidural disease progression. Our approach was to segregate the spinal canal into sectors according to ISRC guidelines ${ }^{4}$ (Fig. 2), in order to anatomically specify the location of epidural disease and evaluate both preoperative and postoperative MR images. In an effort to guide CTV delineation practice, our aim was to determine predictive factors of epidural disease progression following postoperative SBRT, given that no standardizations or recommendations to date have been reported for this indication. The clinical outcomes for this cohort have been previously reported. ${ }^{1}$

In the context of those treated targets (cases) with preoperative epidural disease involving both the posterior and anterior compartments ( $\mathrm{n}=15$, any combination of Sectors 3,4 , or 5 , with 1,2 , or 6 ), epidural disease progression was observed in both the posterior and anterior compartments without sector sparing (Table 2, Fig. 3). The anterior compartment was involved in 11 of $15(73.3 \%)$ cases, and in the posterior compartment in 14 of $15(93.3 \%)$ cases. 
When preoperative (in conjunction with postoperative) epidural disease was confined to the anterior epidural sectors ( $\mathrm{n}=9$ of 24 cases with any combination of Sectors 1, 2, or 6; Table 2), the proportion of cases with posterior compartment epidural failure was significantly lower ( $p$ $=0.047$ ) than the proportion in cases with preoperative epidural disease involving both the anterior and posterior compartments ( $n=15$ of 24 cases; Table 2). Furthermore, among cases with preoperative epidural disease confined to the anterior compartment, when failure was observed in the posterior compartments (Sectors 3 and/or 5), there was selective sparing of Sector 4 . Of note, Sector 4 is anatomically diametrically opposed to Sector 1 (the most anterior sector) (Table 2; Fig. 3). In contrast, we observed that the postoperative MRI epidural disease characteristics (location and Bilsky grade of epidural disease), as well as the total (cumulative) number of ISRC sectors involved (preor postoperatively), were not correlated with the location of epidural failure. Therefore, the sector involvement pattern on preoperative MRI, in conjunction with the postoperative MRI findings, has potential implications for practice, and from our observations we can suggest the following recommendations.

First, we recommend careful evaluation of preoperative spine MRI when planning treatment for these cases. The postoperative MRI alone is insufficient when determining if an epidural sector-sparing CTV versus a donuttype CTV is to be applied. Second, an epidural-sparing horseshoe-type CTV (covering all sectors except Sector 4; Fig. 1) may be appropriate only for those patients with anterior epidural disease involvement alone on both the preoperative and postoperative MRI, with the caveat that no other soft-tissue or bony disease lies in the sector to be treated. Furthermore, this type of CTV likely represents a minimum CTV when considering that failures within the posterolateral epidural spaces were still observed (Sectors 3 and 5; Table 2). This recommendation is based on the lack of involvement of Sector 4 upon progression in these cases. When the anterior and posterior compartments are involved preoperatively, then a donut distribution can be recommended (Fig. 1). This is based on our observations that we did not observe any epidural sector-sparing pattern upon progression (Table 2; Fig. 3). In particular, even though Sector 4 was not involved postoperatively, preoperative involvement was observed in $26.7 \%$ of cases and epidural disease progression was observed in Sector 4 in $29 \%$ of cases. However, we cannot comment on the recommended CTV when only posterior epidural disease is present (i.e., no involvement of the anterior epidural space), as we did not have any such cases. Of note, isolated posterior segment disease in patients with spinal metastases represents the minority of all spine metastases at presentation. ${ }^{2}$ Therefore, we make our recommendations for those cases with anterior epidural sector involvement alone, or anterior with posterior epidural sector involvement, based on the preoperative MRI (in conjunction with our postoperative findings).

Our observations have to be taken in context regarding our spine SBRT CTV approach. Our typical practice was to be overgenerous with respect to covering the epidural space. Almost all cases were treated with a donut distribution (91\%), and almost all observed cases of failure had been treated with a donut distribution (92\%). With a donut distribution, all relevant sectors would have been included in the high-dose volume (Fig. 1). In the 2 cases of failure treated with a horseshoe distribution, both cases exhibited local disease progression confined to the anterior compartment. Hence, these cases do not impact our observations. Therefore, our suggestion of a horseshoe distribution for those cases with anterior epidural disease involvement alone (Fig. 1) on preoperative and postoperative MRI is based on the lack of epidural disease progression in Sector 4 and assumes that we did not need to include that sector initially in the CTV. We believe this is a reasonable assumption given our observations. Furthermore, this recommendation is based on the caveat that no other softtissue or bony disease lies in the sector to be treated. With respect to craniocaudal extension of local failure, we did not observe spread along this direction that was specifically beyond the CTV and PTV. This may represent our approach to also allow for at least 5-mm margins along this axis in our CTV. Lastly, epidural disease progression is likely a consequence of 3 potential factors. The first factor is a potential lack of coverage in the CTV, and this is not the case in our series as almost all cases were treated with an initial donut distribution. The second factor is potential aggressive biology, such that these patients had biologically aggressive disease which rendered them with epidural disease at baseline in almost all cases (96.2\%) and, despite surgery, treatment in these patients failed with the occurrence of epidural disease progression. Whether or not there is distinct biologic differentiation of epidural soft-tissue disease as opposed to the bone-confined disease is unknown. Epidural disease progression is a well-established predictor of progression that is consistent with our initial clinical report. ${ }^{1}$ In that report, we also observed that downgrading epidural disease has therapeutic potential, and in this report we observed that it does not necessarily impact the anatomical pattern of progression. In fact, we report that the initial anatomy of epidural involvement is critical to where progression will occur in those patients who experience progression after spine SBRT. The third factor reflects the dose delivered within the epidural space, and we have used the critical neural tissue (which represents the spinal cord and/or thecal sac) dose as the surrogate. In our initial report, we did not observe that the critical neural tissue dose was a predictive factor in these patients. This may reflect that our maximum point volume doses administered to the critical neural tissue-which are based on published evidence-based recommendations ${ }^{12,14}$-are sufficient and represent a balance between efficacy and safety. However, we cannot comment on whether the number of epidural disease progression cases could have been reduced if we escalated the dose delivered to the critical neural tissue beyond our institutional practice. Ultimately, the data in this report are the most representative of this cohort and spine SBRT practice, and we believe the observed anatomical relationship between the location of preoperative epidural disease and site of progression is generalizable for contouring recommendations.

\section{Conclusions}

We report a detailed pattern-of-failure analysis of epi- 
dural disease progression based on a simple and previously described anatomical classification scheme. Our results support a detailed imaging review of the preoperative epidural disease characteristics, in addition to the postoperative MRI epidural disease characteristics, when planning the CTV. A donut distribution may not be needed when epidural disease is confined pre- and postoperatively to the anterior epidural sectors. Larger-scale analyses are required to validate our observations; however, at this time, these data represent a guide to assist in postoperative spine SBRT CTV contouring.

\section{References}

1. Al-Omair A, Masucci L, Masson-Cote L, Campbell M, Atenafu EG, Parent A, et al: Surgical resection of epidural disease improves local control following postoperative spine stereotactic body radiotherapy. Neuro Oncol 15:1413-1419, 2013

2. Algra PR, Heimans JJ, Valk J, Nauta JJ, Lachniet M, Van Kooten B: Do metastases in vertebrae begin in the body or the pedicles? Imaging study in 45 patients. AJR Am J Roentgenol 158:1275-1279, 1992

3. Bilsky MH, Laufer I, Fourney DR, Groff M, Schmidt MH, Varga PP, et al: Reliability analysis of the epidural spinal cord compression scale. J Neurosurg Spine 13:324-328, 2010

4. Cox BW, Spratt DE, Lovelock M, Bilsky MH, Lis E, Ryu S, et al: International Spine Radiosurgery Consortium consensus guidelines for target volume definition in spinal stereotactic radiosurgery. Int J Radiat Oncol Biol Phys 83:e597e605, 2012

5. Klekamp J, Samii H: Surgical results for spinal metastases. Acta Neurochir (Wien) 140:957-967, 1998

6. Laufer I, Iorgulescu JB, Chapman T, Lis E, Shi W, Zhang Z, et al: Local disease control for spinal metastases following "separation surgery" and adjuvant hypofractionated or highdose single-fraction stereotactic radiosurgery: outcome analysis in 186 patients. J Neurosurg Spine 18:207-214, 2013

7. Massicotte E, Foote M, Reddy R, Sahgal A: Minimal access spine surgery (MASS) for decompression and stabilization performed as an out-patient procedure for metastatic spinal tumours followed by spine stereotactic body radiotherapy (SBRT): first report of technique and preliminary outcomes. Technol Cancer Res Treat 11:15-25, 2012

8. Mizumoto M, Harada H, Asakura H, Hashimoto T, Furutani $\mathrm{K}$, Hashii H, et al: Radiotherapy for patients with metastases to the spinal column: a review of 603 patients at Shizuoka Cancer Center Hospital. Int J Radiat Oncol Biol Phys 79:208-213, 2011

9. Pepe MS, Mori M: Kaplan-Meier, marginal or conditional probability curves in summarizing competing risks failure time data? Stat Med 12:737-751, 1993
10. Sahgal A, Bilsky M, Chang EL, Ma L, Yamada Y, Rhines $\mathrm{LD}$, et al: Stereotactic body radiotherapy for spinal metastases: current status, with a focus on its application in the postoperative patient. J Neurosurg Spine 14:151-166, 2011

11. Sahgal A, Larson DA, Chang EL: Stereotactic body radiosurgery for spinal metastases: a critical review. Int J Radiat Oncol Biol Phys 71:652-665, 2008

12. Sahgal A, Ma L, Weinberg V, Gibbs IC, Chao S, Chang UK, et al: Reirradiation human spinal cord tolerance for stereotactic body radiotherapy. Int J Radiat Oncol Biol Phys 82:107-116, 2012

13. Sahgal A, Roberge D, Schellenberg D, Purdie TG, Swaminath A, Pantarotto J, et al: The Canadian Association of Radiation Oncology scope of practice guidelines for lung, liver and spine stereotactic body radiotherapy. Clin Oncol (R Coll Radiol) 24:629-639, 2012

14. Sahgal A, Weinberg V, Ma L, Chang E, Chao S, Muacevic A, et al: Probabilities of radiation myelopathy specific to stereotactic body radiation therapy to guide safe practice. Int J Radiat Oncol Biol Phys 85:341-347, 2013

15. Thibault I, Al-Omair A, Masucci GL, Masson-Côté L, Lochray F, Korol R, et al: Spine stereotactic body radiotherapy for renal cell cancer spinal metastases: analysis of outcomes and risk of vertebral compression fracture. J Neurosurg Spine 21:711-718, 2014

16. Wang XS, Rhines LD, Shiu AS, Yang JN, Selek U, Gning I, et al: Stereotactic body radiation therapy for management of spinal metastases in patients without spinal cord compression: a phase 1-2 trial. Lancet Oncol 13:395-402, 2012

\section{Disclosures}

Dr. Arjun Sahgal reports having received honoraria for previous educational seminars and research funding from Elekta $\mathrm{AB}$ and honorarium from Medtronic for educational seminars.

\section{Author Contributions}

Conception and design: Sahgal, Chan. Acquisition of data: Sahgal, Chan, Thibault, Yu, Letourneau, Lee. Analysis and interpretation of data: all authors. Drafting the article: all authors. Critically revising the article: all authors. Reviewed submitted version of manuscript: all authors. Approved the final version of the manuscript on behalf of all authors: Sahgal. Statistical analysis: Sahgal, Atenafu.

\section{Correspondence}

Arjun Sahgal, Department of Radiation Oncology, Sunnybrook Health Sciences Centre, University of Toronto, 2075 Bayview Ave., Toronto, ON M4N 3M5, Canada. email: arjun.sahgal@ sunnybrook.ca. 